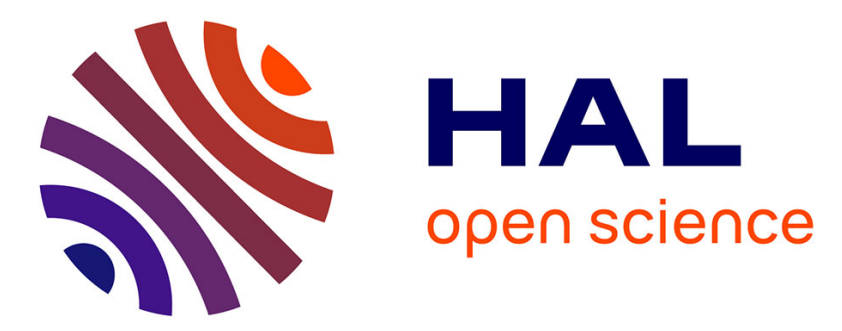

\title{
Homogeneous continuous finite-time observer for the triple integrator
}

\author{
Emmanuel Bernuau, Denis Efimov, Emmanuel Moulay, Wilfrid Perruquetti
}

\section{To cite this version:}

Emmanuel Bernuau, Denis Efimov, Emmanuel Moulay, Wilfrid Perruquetti. Homogeneous continuous finite-time observer for the triple integrator. ECC'15 - 14th annual European Control Conference, Jul 2015, Linz, Austria. hal-01140339

\section{HAL Id: hal-01140339 \\ https://inria.hal.science/hal-01140339}

Submitted on 8 Apr 2015

HAL is a multi-disciplinary open access archive for the deposit and dissemination of scientific research documents, whether they are published or not. The documents may come from teaching and research institutions in France or abroad, or from public or private research centers.
L'archive ouverte pluridisciplinaire HAL, est destinée au dépôt et à la diffusion de documents scientifiques de niveau recherche, publiés ou non, émanant des établissements d'enseignement et de recherche français ou étrangers, des laboratoires publics ou privés. 


\title{
Homogeneous continuous finite-time observer for the triple integrator
}

\author{
Emmanuel Bernuau, Denis Efimov, Emmanuel Moulay and Wilfrid Perruquetti*
}

\begin{abstract}
In this paper we consider the continuous homogeneous observer defined in [1] in the case of the triple integrator. In [1], convergence of the algorithm was only proved when the degree of homogeneity was sufficiently close to 0 without more tractable information. We show here that, in the case of the triple integrator, the observer presents global finite-time stability for any negative degree under constructive conditions on the gains. This is achieved with a homogeneous Lyapunov function design. Simulations of the proposed observer are also provided.
\end{abstract}

\section{Introduction}

Even though mechanical or electrical systems often have a second order model, problems where third order systems appear are also common and the simplest and canonical form of these systems is a triple integrator. Most of the current techniques for linear or nonlinear feedback stabilization and observation provide an asymptotic or exponential stability: the obtained closed-loop dynamics is locally Lipschitz and the system trajectories settle at the origin when the time is approaching infinity. Such a rate of convergence is not admissible in many applications, this is why the FiniteTime Stability (FTS) notion has been quickly developing during the last decades: solutions of a FTS system reach the equilibrium point in a finite time. For example, for $x \in \mathbb{R}$ and $\alpha \in(0,1)$, the solutions of

${ }^{*}$ E. Bernuau is with IRCCyN, UMR CNRS 6597, Ecole centrale de Nantes, France; D. Efimov and W. Perruquetti are with Non-A team, Inria Nord de France, France and CRIStAL, UMR CNRS 9189, Ecole Centrale de Lille, France; D. Efimov is with Department of Control Systems and Informatics, Saint Petersburg State University of Information Technologies Mechanics and Optics (ITMO), Russia; E. Moulay is with XLIM-SIC, UMR CNRS 7252, Université de Poitiers, France. This work was supported in part by the Government of Russian Federation (Grant 074-U01) and the Ministry of Education and Science of Russian Federation (Project 14.Z50.31.0031) and by the ANR funded project Qualiphe.

$$
\begin{aligned}
& \dot{x}=-\operatorname{sign}(x)|x|^{\alpha} \text { starting from } x_{0} \in \mathbb{R} \text { at } t_{0}=0 \text { are } \\
& \left\{\begin{array}{lll}
\operatorname{sign}\left(x_{0}\right)\left[\left|x_{0}\right|^{1-\alpha}-(1-\alpha) t\right]^{\frac{1}{1-\alpha}} & \text { if } \quad 0 \leq t \leq \frac{\left|x_{0}\right|^{1-\alpha}}{1-\alpha} \\
0 & \text { if } t>\frac{\left|x_{0}\right|^{1-\alpha}}{1-\alpha}
\end{array} .\right.
\end{aligned}
$$

Let us note that the right hand side of the above differential equation is not Lipschitz. In fact, finite-time convergence implies non-uniqueness of solutions (in backward time) which is not possible in the presence of Lipschitzcontinuous dynamics, where different maximal trajectories never cross.

The problem of finite-time stability has been developed for continuous systems giving sufficient and necessary condition (see $[2,3]$ ). In addition, necessary and sufficient conditions appear for discontinuous systems (see [4]). It was observed in many papers that FTS can be achieved if the system is locally asymptotically stable and homogeneous with negative degree [5]. This is why the homogeneity plays a central role in the FTS system design. The reader may found additional properties and results on homogeneity in $[6,7,8,9,10]$. The homogeneity property was used many times to design FTS state controls [11, 12, 13, 14, 15, 16], FTS observers [1, 17, 18], consensus protocols [19] and FTS output feedback [20, 21].

In [1], a generic class of homogeneous continuous observers for an $n-t h$ integrator was developed. Their simple shape, combined with FTS and robustness properties granted by homogeneity were making them very promising candidates for observation. However, the proof of stability relied on a continuity argument and the result only held when the homogeneity degree was sufficiently close to 0 , i.e., when the observer was almost linear. No constructive procedure was known, given a degree of homogeneity, to check whether the observer was stable or not, for $n>2$.

In this paper, we shall give explicit conditions under which the result of [1] holds in the case of the triple integrator. The paper is organized as follows. Section II is devoted to preliminaries. In section III we present the system of interest, the proposed observer and the corresponding error equation. In section IV, we introduce our 
candidate Lyapunov function and give conditions under which this function is positive definite. The derivative of this function is studied in section $\mathrm{V}$, and the main results are compiled in theorems 1 and 2. Simulations are presented in section VI and finally a conclusion summarizes the paper.

\section{Preliminaries}

Through the paper the following notation will be used. For any real number $\alpha \geq 0$ and for all $x \in \mathbb{R}$ we define $\lfloor x\rceil^{\alpha}=\operatorname{sign}(x)|x|^{\alpha}$.

Proposition 1 (Young's inequality). For any $x, y \in \mathbb{R}$, any $p, q>0$ such that $\frac{1}{p}+\frac{1}{q}=1$ and any $\varepsilon>0$, the following inequality holds

$$
|x y| \leq \varepsilon \frac{|x|^{p}}{p}+\frac{1}{\varepsilon} \frac{|y|^{q}}{q} .
$$

This well known inequality will be used extensively throughout the paper.

\subsection{Finite-time stabilization}

Let us consider a continuous vector field $f$ and the system

$$
\dot{x}=f(x), \quad x \in \mathbb{R}^{n} .
$$

Definition 1. [22] The origin of the system (1) is finitetime stable (FTS) iff there exists a neighborhood of the origin $\mathscr{V}$ such that:

1. For any $x_{0} \in \mathscr{V}$ there exists $t_{0} \geq 0$ such that for any solution $x(t)$ of (1) such that $x(0)=x_{0}$ we have $x(t)=0$ for all $t \geq t_{0}$. The infimum $T\left(x_{0}\right)$ of all such $t_{0}$ allows us to define the function $T: \mathscr{V} \rightarrow$ $\mathbb{R}_{+}$, called the settling-time function of the system (1).

2. For any neighborhood of the origin $\mathscr{U}_{1} \subset \mathscr{V}$, there exists a neighborhood of the origin $\mathscr{U}_{2}$ such that for any $x_{0} \in \mathscr{U}_{2}$ and any solution $x(t)$ of (1) such that $x(0)=x_{0}$ we have $x(t) \in \mathscr{U}_{1}$ for all $t \geq 0$.

Moreover, if the neighborhood $\mathscr{V}$ can be chosen to be $\mathbb{R}^{n}$, then the origin of the system (1) is said to be globally finite-time stable (GFTS).

\subsection{Homogeneity}

Let $\mathbf{r}=\left(r_{1}, \ldots, r_{n}\right)$ be a $n$-uplet of positive real numbers, thereafter called a generalized weight. Then $\Lambda_{r} x=\left(\ldots, \lambda^{r_{i}} x_{i}, \ldots\right)$ for any positive number $\lambda$ represents a mapping $x \mapsto \Lambda_{\mathbf{r}} x$ usually called a dilation (see [8]).
Definition 2. A function $h: \mathbb{R}^{n} \rightarrow \mathbb{R}$ is $\mathbf{r}$-homogeneous of degree $\kappa \in \mathbb{R}$ if for all $x \in \mathbb{R}^{n}$ and all $\lambda>0$ we have $h\left(\Lambda_{\mathbf{r}} x\right)=\lambda^{\kappa} h(x)$.

Definition 3. A vector field $f: \mathbb{R}^{n} \rightarrow \mathbb{R}^{n}$ is rhomogeneous of degree $\kappa$ if for all $x \in \mathbb{R}^{n}$ and all $\lambda>0$ we have $f\left(\Lambda_{r} x\right)=\lambda^{\kappa} \Lambda_{r} f(x)$, or equivalently, if the coordinate functions $f_{i}$ are $\mathbf{r}$-homogeneous of degree $\kappa+r_{i}$. When such a property holds, the corresponding system (1) is said to be $\mathbf{r}$-homogeneous of degree $\kappa$.

\section{Problem formulation}

Consider the following system defined for $z=$ $\left(z_{1}, z_{2}, z_{3}\right)^{T} \in \mathbb{R}^{3}$

$$
\left\{\begin{array}{l}
\dot{z}_{1}=z_{2} \\
\dot{z}_{2}=z_{3} \\
\dot{z}_{3}=u \\
y=z_{1}
\end{array}\right.
$$

where $z_{1}, z_{2}$ and $z_{3}$ are the states of the system, $u$ is the input and $y$ is the output. We follow [1] and define, for $\beta \in\left(\frac{2}{3}, 1\right)$, the following estimator

$$
\left\{\begin{array}{l}
\dot{\hat{z}}_{1}=\hat{z}_{2}+l_{1}\left\lfloor y-\hat{z}_{1}\right\rceil^{\beta} \\
\dot{\hat{z}}_{2}=\hat{z}_{3}+l_{2}\left\lfloor y-\hat{z}_{1}\right\rceil^{2 \beta-1} \\
\dot{\hat{z}}_{3}=u+l_{3}\left\lfloor y-\hat{z}_{1}\right\rceil^{3 \beta-2}
\end{array} .\right.
$$

Consider now the estimation error $x=z-\hat{z}$. Its dynamics is given by

$$
\left\{\begin{array}{l}
\dot{x}_{1}=x_{2}-l_{1}\left\lfloor x_{1}\right\rceil^{\beta} \\
\dot{x}_{2}=x_{3}-l_{2}\left\lfloor x_{1}\right\rceil^{2 \beta-1} \\
\dot{x}_{3}=-l_{3}\left\lfloor x_{1}\right\rceil^{3 \beta-2}
\end{array} .\right.
$$

We intend to find explicit conditions on the gains $l_{1}, l_{2}$ and $l_{3}$ and on the power $\beta$ such that the origin is a GFTS equilibrium of the system (4).

Remark 1. The right-hand side of system (4) is not Lipschitz continuous. Even though we could select $\beta \geq 1$ to ensure Lipschitz continuity, we would lose the finitetime stability which is one of the desired properties of the algorithm. Therefore, we will stick to $\beta<1$.

\section{Lyapunov function design}

Let us define, for $\rho, \delta>0$, the following candidate Lyapunov's function

$$
\begin{aligned}
V(x) & =\left(l_{2}-\beta \rho l_{3}\right) \frac{\left|x_{1}\right|^{2 \beta}}{2 \beta}-\rho l_{3}\left\lfloor x_{1}\right\rceil^{\beta} x_{2}+\frac{x_{2}^{2}}{2} \\
& -\frac{l_{1}}{l_{2}} x_{2}\left\lfloor x_{3}\right\rceil^{\frac{\beta}{2 \beta-1}}+\delta \frac{2 \beta-1}{2 \beta}\left|x_{3}\right|^{\frac{2 \beta}{2 \beta-1}}-x_{1} x_{3} .
\end{aligned}
$$


Using the Young's inequality, we find that $V$ is definite positive if the following inequalities hold

$$
\begin{aligned}
l_{2}-\beta \rho l_{3}-\varepsilon_{1} \rho l_{3} \beta-\varepsilon_{3} & >0 \\
1-\frac{\rho l_{3}}{\varepsilon_{1}}-\frac{l_{1} \varepsilon_{2}}{l_{2}} & >0 \\
\delta(2 \beta-1)-\frac{l_{1} \beta}{l_{2} \varepsilon_{2}}-\frac{2 \beta-1}{\varepsilon_{3}} & >0
\end{aligned}
$$

We claim that, under the two following conditions, we can find $\varepsilon_{1}, \varepsilon_{2}$ and $\varepsilon_{3}$ such that (6), (7) and (8) hold

$$
\begin{gathered}
l_{2}-\beta \rho l_{3}-\beta \rho^{2} l_{3}^{2}>0 \\
\delta>\frac{\beta l_{1}^{2} l_{2}-\beta^{2} \rho l_{3} l_{1}^{2}+2 \sqrt{2 \beta-1} \beta \rho l_{1} l_{2} l_{3}+(2 \beta-1) l_{2}^{2}}{(2 \beta-1) l_{2}^{2}\left(l_{2}-\beta \rho l_{3}-\beta \rho^{2} l_{3}^{2}\right)} .
\end{gathered}
$$

Proposition 2. Under conditions (9) and (10), the function $V$ defined by (5) is positive definite.

The proof is omitted due to space limitations.

Let us remark that the conditions (9) and (10) do not really constrain the gains. Indeed, $\rho$ and $\delta$ are free parameters appearing only in the Lyapunov function $V$ and taking $\rho$ small enough ensures condition (9) while taking $\delta$ large enough ensures condition (10).

\section{Study of the derivative of $V$}

Our goal in this section is to prove that an adequate choice of the parameters $\delta$ and $\rho$ together with conditions on the gains $l_{1}, l_{2}$ and $l_{3}$ can ensure that the derivative of $V$ will be negative definite.

Let us start by mentioning that the function $V$ is not differentiable on the plane $x_{1}=0$. It will not be a big deal because we will see that $\dot{V}$ is upper bounded by $-C V^{(\beta+1) /(2 \beta)}$ with $C>0$ and because the only invariant subspace of the plane $x_{1}=0$ is $\{0\}$. However, to ensure the correctness of the following computations, we assume that $x_{1} \neq 0$. A direct computation gives

$$
\begin{aligned}
\dot{V}(x) & =\left(\rho l_{3} l_{2}+l_{3}+l_{1} \beta \rho l_{3}-l_{1} l_{2}\right)\left|x_{1}\right|^{3 \beta-1} \\
-\beta & \rho l_{3}\left|x_{1}\right|^{\beta-1} x_{2}^{2}-\frac{l_{1}}{l_{2}}\left|x_{3}\right|^{\frac{3 \beta-1}{2 \beta-1}} \\
+ & \rho l_{2} \beta\left(l_{1}-1\right)\left\lfloor x_{1}\right\rceil^{2 \beta-1} x_{2}+\left(l_{1}-\rho l_{3}\right)\left\lfloor x_{1}\right\rceil^{\beta} x_{3} \\
& +l_{1}\left\lfloor x_{1}\right\rceil^{2 \beta-1}\left\lfloor x_{3}\right\rceil^{\frac{\beta}{2 \beta-1}}-\delta l_{3}\left\lfloor x_{1}\right]^{3 \beta-2}\left\lfloor x_{3}\right\rceil^{\frac{1}{2 \beta-1}} \\
& +\quad \frac{l_{1} l_{3} \beta}{l_{2}(2 \beta-1)}\left\lfloor x_{1}\right\rceil^{3 \beta-2} x_{2}\left\lfloor x_{3}\right\rceil^{\frac{1-\beta}{2 \beta-1}} .
\end{aligned}
$$

From successive uses of the Young's inequality, we find that $\dot{V}(x)<0$ on $\left\{x_{1} \neq 0\right\}$ if there exist $\varepsilon_{1}, \varepsilon_{2}, \varepsilon_{3}>0$ such that the following conditions hold

$$
l_{1}>\rho l_{3}
$$

$$
\begin{gathered}
l_{1}\left[-(3 \beta-1) l_{2}+\beta \varepsilon_{2}+\varepsilon_{3}(2 \beta-1)\right] \\
+l_{3}\left[(3 \beta-1)\left(\rho l_{2}+1+l_{1} \beta \rho+\frac{\rho \beta\left|l_{1}-1\right| \varepsilon_{1}}{2}\right)\right. \\
\left.-\rho \beta \varepsilon_{2}+\delta(3 \beta-2)+\frac{l_{1}}{l_{2}} \frac{\beta(5 \beta-3)}{2(2 \beta-1)}\right]<0 \\
\quad \rho\left[\frac{\left|l_{1}-1\right|}{\varepsilon_{1}}-2\right]+\frac{l_{1}}{l_{2}(2 \beta-1)}<0 \\
l_{1}\left[-\frac{3 \beta-1}{l_{2}}+\frac{2 \beta-1}{\varepsilon_{2}}+\frac{\beta}{\varepsilon_{3}}\right] \\
+l_{3}\left[-\frac{\rho(2 \beta-1)}{\varepsilon_{2}}+\delta+\frac{l_{1} \beta(1-\beta)}{l_{2}(2 \beta-1)}\right]<0 .
\end{gathered}
$$

There exists $\varepsilon_{1}>0$ such that inequation (13) holds if and only if

$$
-2 \rho+\frac{l_{1}}{l_{2}(2 \beta-1)}<0
$$

and then $\varepsilon_{1}$ has to be chosen such that

$$
\varepsilon_{1}>\frac{\left|l_{1}-1\right| l_{2} \rho(2 \beta-1)}{2 l_{2} \rho(2 \beta-1)-l_{1}} .
$$

Hence, there exist solutions $\left(\varepsilon_{1}, \varepsilon_{2}, \varepsilon_{3}\right)$ of inequalities (12) - (14) if and only if there exist solutions $\left(\varepsilon_{2}, \varepsilon_{3}\right)$ of

$$
\begin{aligned}
l_{1}[ & \left.-(3 \beta-1) l_{2}+\beta \varepsilon_{2}+\varepsilon_{3}(2 \beta-1)\right] \\
+l_{3}[ & {\left[(3 \beta-1)\left(\rho l_{2}+1+l_{1} \beta \rho+\frac{\rho^{2} \beta\left|l_{1}-1\right|^{2} l_{2}(2 \beta-1)}{4 l_{2} \rho(2 \beta-1)-2 l_{1}}\right)\right.} \\
& \left.-\rho \beta \varepsilon_{2}+\delta(3 \beta-2)+\frac{l_{1}}{l_{2}} \frac{\beta(5 \beta-3)}{2(2 \beta-1)}\right]<0, \quad(17)
\end{aligned}
$$

together with (11), (14) and (15). Now denoting

$$
\begin{aligned}
A_{1}= & -(3 \beta-1) l_{2}+\beta \varepsilon_{2}+(2 \beta-1) \varepsilon_{3} \\
A_{3}= & (3 \beta-1)\left(\rho l_{2}+1+l_{1} \beta \rho+\frac{\rho^{2} \beta\left|l_{1}-1\right|^{2} l_{2}(2 \beta-1)}{4 l_{2} \rho(2 \beta-1)-2 l_{1}}\right) \\
& -\rho \beta \varepsilon_{2}+\delta(3 \beta-2)+\frac{l_{1}}{l_{2}} \frac{\beta(5 \beta-3)}{2(2 \beta-1)} \\
B_{1}= & -\frac{3 \beta-1}{l_{2}}+\frac{2 \beta-1}{\varepsilon_{2}}+\frac{\beta}{\varepsilon_{3}} \\
B_{3}= & -\frac{\rho(2 \beta-1)}{\varepsilon_{2}}+\delta+\frac{l_{1} \beta(1-\beta)}{l_{2}(2 \beta-1)}
\end{aligned}
$$

we see that (17) and (14) can be written $A_{1} l_{1}+A_{3} l_{3}<0$, $B_{1} l_{1}+B_{3} l_{3}<0$. Hence, the negativity of $\dot{V}$ would be ensured for small enough $l_{3}$ if we were able to prove that the terms $A_{1}$ and $B_{1}$ are negative.

Lemma 1. For any $\beta \in(2 / 3,1)$ and any $l_{2}>0$ there exists a pair $\left(\varepsilon_{2}, \varepsilon_{3}\right)$ of positive reals such that

$$
\left\{\begin{array}{ll}
\beta \varepsilon_{2}+(2 \beta-1) \varepsilon_{3} & <l_{2}(3 \beta-1) \\
\frac{2 \beta-1}{\varepsilon_{2}}+\frac{\beta}{\varepsilon_{3}} & <\frac{3 \beta-1}{l_{2}}
\end{array} .\right.
$$


The proof is omitted due to space limitations.

Finally we can state our main result.

Theorem 1. For any $\beta \in(2 / 3,1)$, any $l_{1}, l_{2}>0$ and any $\rho$ and $\delta$ such that

$$
\begin{gathered}
\rho>\frac{l_{1}}{2(2 \beta-1) l_{2}} \\
\delta>\frac{\beta l_{1}^{2}+(2 \beta-1) l_{2}}{(2 \beta-1) l_{2}^{2}}
\end{gathered}
$$

there exist $l_{3}>0$ such that $V$ is positive definite and $\dot{V}(x)<0$ for all $x \in\left\{x_{1} \neq 0\right\}$.

Proof. From all the discussion beforehand, it suffices to find positive reals $l_{3}, \varepsilon_{2}$ and $\varepsilon_{3}$ such that the inequalities (9), (10), (11), (14), (15) and (17) hold.

Clearly, the condition (19) is just a rewritting of inequality (15). Inequality (9) is equivalent to

$$
l_{3}<\frac{1}{2 \rho}\left[\sqrt{1+\frac{4 l_{2}}{\beta}}-1\right] .
$$

Condition (20) ensures that inequality (10) has solutions. Inequality (10) can then be rewritten under the form

$$
l_{3}<\kappa\left(l_{1}, l_{2}, \rho, \delta\right) .
$$

An explicit form of the function $\kappa$ can be computed but is not presented here due to space limitations.

Inequality (11) can be rewritten

$$
l_{3}<\frac{l_{1}}{\rho} .
$$

Now, granted that we choose $\varepsilon_{2}$ and $\varepsilon_{3}$ for which (18) holds, we see that $A_{3}>0$ and then inequalities (17) and (14) can be rewritten

$$
\begin{gathered}
l_{3}<-\frac{A_{1}}{A_{3}} l_{1} \\
B_{3} \leq 0 \quad \text { or } \quad l_{3}<-\frac{B_{1}}{B_{3}} l_{1} .
\end{gathered}
$$

Finally, by lemma 1 , we get $A_{1}<0$ and $B_{1}<0$ and all inequalities (21) - (25) hold if $l_{3}$ is chosen small enough, which concludes the proof.

Let us remark that, although this theorem seems to only state the existence of a gain $l_{3}>0$ such that the Lyapunov function $V$ is positive definite and its derivative $\dot{V}$ is negative for $x_{1} \neq 0$, it is actually constructive. To compute admissible values of $l_{3}>0$, it suffices to

1. select $\beta \in(2 / 3,1), l_{1}, l_{2}>0, \rho$ and $\delta$ such that (19) and (20) hold;
2. compute a couple $\left(\varepsilon_{2}, \varepsilon_{3}\right)$ such that (18) holds;

3. select $l_{3}$ such that all inequalities (21) - (25) hold.

Example 1. Let us select $\beta=0.75$ and $l_{1}=l_{2}=1$. Conditions (19) and (20) read $\rho>1$ and $\delta>2.5$ so we choose $\rho=6$ and $\delta=3$. Then we select $\varepsilon_{2}=\frac{5}{6}$ and $\varepsilon_{3}=$ $\frac{6}{5}$ and easily check that (18) holds. Then inequalities (21) - (24) read (numerical values have been truncated)

$$
\begin{aligned}
& l_{3} \leq 0.126 \\
& l_{3} \leq 0.145 \\
& l_{3} \leq 0.166 \\
& l_{3} \leq 0.002
\end{aligned}
$$

while inequality (25) trivially holds because $B_{3}<0$. The choice $l_{3}=0.002$ then ensures that $V$ is positive definite and $\dot{V}<0$ for $x_{1} \neq 0$.

We can now express the result in a more theoretical form.

Theorem 2. For any $\beta \in(2 / 3,1)$, for any $l_{1}>0$ and $l_{2}>0$, there exists $l_{3}>0$ such that the observer (3) recovers in finite time the state $z$ of the system (2) for any initial state $z_{0} \in \mathbb{R}^{3}$.

Proof. We need to prove that the origin is a GFTS equilibrium for the error equation (4). Let us select $\rho, \delta, \varepsilon_{2}, \varepsilon_{3}$ and $l_{3}$ such that inequalities (19), (20), (18) and (21) - (25) hold.

Let us denote $-a=\sup \left\{\dot{V}(x): V(x)=1, x_{1} \neq 0\right\}$. Given that $\dot{V}<0$ on $\left\{V(x)=1, x_{1} \neq 0\right\}$, we have $a \geq 0$. Moreover, $\{V(x)=1\}$ is compact: indeed, any continuous homogeneous function is proper [11] and a straightforward computation shows that $V$ and $\dot{V}$ are $(1, \beta, 2 \beta-1)$-homogeneous functions of degree $2 \beta$ and $3 \beta-1$. Since $0 \notin\{V(x)=1\}$, we get $a>0$. Classical manipulations on homogeneous functions then show that for any $\left\{x \in \mathbb{R}^{3}: x_{1} \neq 0\right\}$ we have

$$
\dot{V}(x) \leq-a V(x)^{(3 \beta-1) /(2 \beta)} .
$$

Now, given that no solution of (4), except $x(t)=0$, stays on $\left\{x_{1}=0\right\}$, the set $\{V \leq 1\}$ is strictly positively invariant and therefore the origin is globally asymptotically stable for system (4) [11]. But system (4) is $(1, \beta, 2 \beta-1)$-homogeneous of degree $\beta-1<0$, and therefore the origin is GFTS [11].

Sometimes the conditions (21) - (25) may lead to very small values of the gain $l_{3}$. It is possible to rescale it with the following procedure. 
Proposition 3. Set $\beta \in(2 / 3,1)$. If the origin is GFTS for system (4) with a set of gains $\left(l_{1}, l_{2}, l_{3}\right)$, then for any $\lambda>0$ the origin is also GFTS for system (4) with the set of gains $\left(\lambda l_{1}, \lambda^{2} l_{2}, \lambda^{3} l_{3}\right)$.

The proof is omitted due to space limitations.

Remark that, for this new set of gains, corresponding values $\delta, \rho, \varepsilon_{2}$ and $\varepsilon_{3}$ such that all the conditions (20) - (25) hold may not exist or be different, but the finite-time stability is still ensured.

Example 2 (Example 1 continued). We take again $\beta=$ $0.75, \rho=6, \delta=3, \varepsilon_{2}=\frac{5}{6}$ and $\varepsilon_{3}=\frac{6}{5}$. The set of gains $(1,1,0.002)$ is valid, thus by proposition 3 , taking $\lambda=10^{3}$, the set of gains $\left(10^{3}, 10^{6}, 2.10^{6}\right)$ is also valid. However, inequalities (19) and (15) read $\rho>10^{-3}$ and $\rho<5.10^{-4}$ and thus this set of gains could not have been found by the direct method exposed after theorem 1.

A local approach can also be used to extend our result to any linear system with an output of relative degree 3 .

Corollary 1. Consider a linear system of dimension 3 such that the output $y$ is of relative degree 3. The system reads $\dot{x}=A x+B u$ with $x=[y, \dot{y}, \ddot{y}]^{T}, A=$ $\left(\begin{array}{ccc}0 & 1 & 0 \\ 0 & 0 & 1 \\ c_{1} & c_{2} & c_{3}\end{array}\right)$ and $B=[0,0, \lambda]^{T}$ where $c_{1}, c_{2}, c_{3}$ and $\lambda$ are real coefficients. Then, if the gains $l_{i}$ are selected according to the conditions presented before, the following observer leads the origin of the error equation to being locally finite-time stable:

$$
\dot{\hat{x}}=A \hat{x}+B u+\left(\begin{array}{c}
l_{1}\left\lfloor x_{1}-\hat{x}_{1}\right\rceil^{\beta} \\
l_{2}\left\lfloor x_{1}-\hat{x}_{1}\right\rceil^{2 \beta-1} \\
l_{3}\left\lfloor x_{1}-\hat{x}_{1}\right]^{3 \beta-2}
\end{array}\right) .
$$

Proof. Computing a local homogeneous approximation of the error equation leads to system (4) which is finitetime stable. Therefore the original system is locally finite-time stable for gains selected according to the conditions presented before. For details about local homogeneous approximations, see [23].

\section{Examples and Simulations}

In this section, we would like to illustrate the applicability of the proposed algorithm in showing how to use it on concrete examples and demonstrate the efficiency of the proposed observer compared to a Luenberger observer.

Consider an electric motor acting on a rigid arm turning around a central point in the horizontal plane.
Let denote $\theta$ the angle of the arm and $\dot{\theta}$ the angular velocity of the arm. The force generated by the motor is $K i$ where $i$ is the intensity of the current and $K>0$ a constant. We find

$$
\begin{aligned}
m \ddot{\theta} & =F-b \theta \\
u & =L \frac{d i}{d t}+R i+k \dot{\theta}
\end{aligned}
$$

where $u$ is the input tension, $b>0$ is a coefficient of friction, $m$ is the mass of the arm and $L, R$ and $k$ are positive coefficients of the motor. The output is supposed to be $\theta$ and is of relative degree 3 . Following Corollary 1 , we get a locally finite-time stable observer.

Now, we would like to compare the proposed observer with a classical Luenberger observer. As in Example 1 , we take $\beta=0.75, \rho=6, \delta=3, \varepsilon_{2}=\frac{5}{6}$ and $\varepsilon_{3}=\frac{6}{5}$. The set of gains $(1,1,0.002)$ is valid, thus by proposition 3 , taking $\lambda=20$, the set of gains $(20,400,16)$ is also valid. We will compare the estimation error given by (4) with the estimation error

$$
\left\{\begin{array}{l}
\dot{x}_{1}=x_{2}-20 x_{1} \\
\dot{x}_{2}=x_{3}-400 x_{1} \\
\dot{x}_{3}=-16 x_{1}
\end{array}\right.
$$

coming from the following observer of system (2)

$$
\left\{\begin{array}{l}
\dot{\hat{z}}_{1}=\hat{z}_{2}+20\left(y-\hat{z}_{1}\right) \\
\dot{\hat{z}}_{2}=\hat{z}_{3}+400\left(y-\hat{z}_{1}\right) \\
\dot{z}_{3}=u(y)+16\left(y-\hat{z}_{1}\right)
\end{array} .\right.
$$

The results of simulations are presented in Fig. 1. The eigenvalues of the matrix corresponding to the linear system (26) are $-9.980+17.309 i,-9.980-$ $17.309 i$ and -0.040 , ensuring the asymptotic stability. However, the small value of the third eigenvalue does not allow the linear observer to recover quickly the signal. We can see that in about $1.2 s$ the homogeneous observer has converged, while $x_{3}$ is still around -0.2 after 1.6s.

\section{Conclusion}

In this paper, we gave explicit conditions under which the observer (3) converges to the state of the system (2) so that the origin is a GFTS equilibrium of the corresponding error equation (4). We introduced a homogeneous Lyapunov function and gave explicit conditions under which this function is positive definite and its derivative is negative. Examples of use of these conditions were provided, as well as a method to derive other valid choices for the gains. Computer simulations demonstrated the efficiency of the proposed algorithm.

Future works include an optimization of the parameters $\rho$ and $\delta$ to let the gain $l_{3}$ take values as large as 

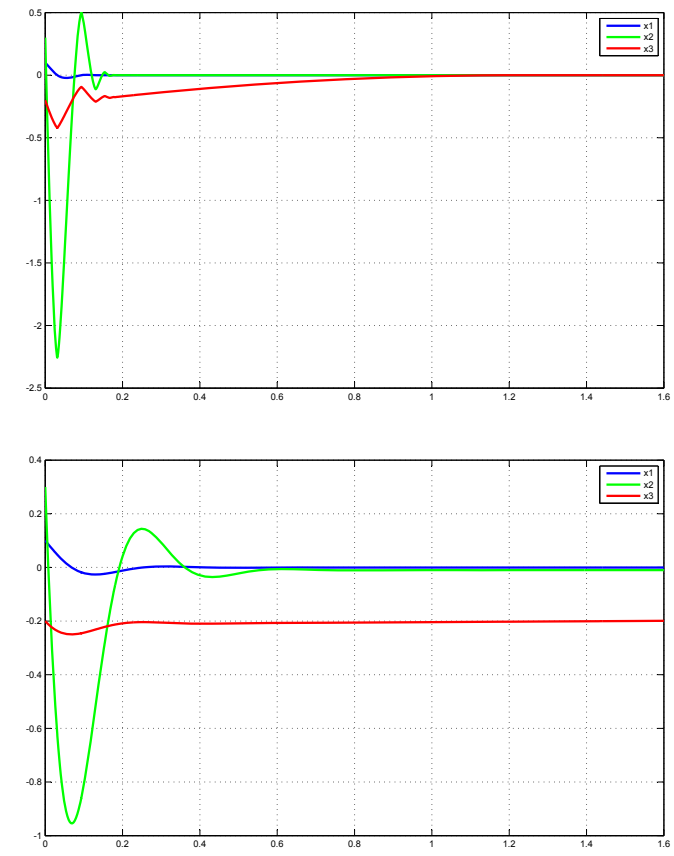

Figure 1. Comparison between homogeneous (top) and linear (bottom) estimation errors.

possible. Also, we would like to go further and try to extend this method to $n^{\text {th }}$-integrators.

\section{References}

[1] W. Perruquetti, T. Floquet, and E. Moulay, "Finitetime observers: application to secure communication," IEEE Transactions on Automatic Control, vol. 53, no. 1, pp. 356-360, 2008.

[2] V. Haimo, "Finite time controllers," SIAM J. Control Optim., vol. 24, no. 4, pp. 760-770, 1986.

[3] E. Moulay and W. Perruquetti, "Finite time stability of non linear systems," in IEEE Conference on Decision and Control, (Hawaii, USA), pp. 3641-3646, 2003.

[4] Y. Orlov, "Finite time stability and robust control synthesis of uncertain switched systems," SIAM J. Control Optim., vol. 43, no. 4, pp. 1253-1271, 2005.

[5] L. Rosier, Etude de Quelques Problèmes de Stabilisation. $\mathrm{PhD}$ thesis, Ecole Normale Supérieure de Cachan, 1993.

[6] A. Bacciotti and L. Rosier, Lyapunov Functions and Stability in Control Theory. Springer, 2nd ed., 2005.

[7] S. Bhat and D. Bernstein, "Finite-time stability of homogeneous systems," in Proceedinds of the American Control Conference, (Albuquerque, USA), pp. 2513-2514, 1997.

[8] H. Hermes, Homogeneous coordinates and continuous asymptotically stabilizing feedback controls, vol. 109 of Differential Equations: Stability and Control, pp. 249260. Marcel Dekker, 1991.

[9] M. Kawski, "Geometric homogeneity and stabilization," in Proc. IFAC Nonlinear Control Symposium (A. Krener and D. Mayne, eds.), (Lake Tahoe, CA), pp. 164-169, 1995.

[10] Y. Orlov, "Finite time stability of homogeneous switched systems," in IEEE Conference on Decision and Control, (Hawaii, USA), pp. 4271-4276, 2003.

[11] S. P. Bhat and D. Bernstein, "Continuous finite-time stabilization of the translational and rotational double integrator," IEEE Trans. Automat. Control, vol. 43, pp. 678682, may 1998.

[12] T. Floquet, J. P. Barbot, and W. Perruquetti, "Higherorder sliding mode stabilization for a class of nonholonomic perturbed systems," Automatica, vol. 39, no. 6, pp. 1077-1083, 2003.

[13] Y. Hong, "Finite-time stabilization and stabilizability of a class of controllable systems," Systems \& Control Letters, vol. 46, no. 4, pp. 231-236, 2002.

[14] E. Moulay and W. Perruquetti, "Finite time stability and stabilization of a class of continuous systems," Journal of Mathematical Analysis and Application, vol. 323, no. 2, pp. 1430-1443, 2006.

[15] C. Qian and W. Lin, "Non-lipschitz continuous stabilizers for nonlinear systems with uncontrollable unstable linearization,' Systems \& Control Letters, vol. 16, no. 3, pp. 441-463, 2001.

[16] L. Praly, "Generalized weighted homogeneity and state dependent time scale for linear controllable systems," in Proc. IEEE CDC 97, (San Diego, California USA), pp. 4342-4347, IEEE, 1997.

[17] Y. Shen and X. Xia, "Semi-global finite-time observers for nonlinear systems," Automatica, vol. 44, no. 12, pp. 3152-3156, 2008.

[18] T. Menard, E. Moulay, and W. Perruquetti, "A global high-gain finite-time observer," IEEE Transactions on Automatic Control, vol. 55, no. 6, pp. 1500-1506, 2010.

[19] Y. Zhang and Y. Yang, "Finite-time consensus of second-order leader-following multi-agent systems without velocity measurements," Physics Letters A, vol. 377, no. 3-4, pp. 243-249, 2013.

[20] Y. Hong, J. Huang, and Y. Xu, "On an output feedback finite-time stabilization problem," IEEE Trans. Automat. Control, vol. 46, pp. 305-309, 2001.

[21] J. Li and C. Qian, "Global finite-time stabilization by dynamic output feedback for a class of continuous nonlinear systems," IEEE Transactions on Automatic Control, vol. 51, no. 5, pp. 879-884, 2006.

[22] S. Bhat and D. Bernstein, "Finite time stability of continuous autonomous systems," SIAM J. Control Optim., vol. 38, no. 3, pp. 751-766, 2000.

[23] V. Andrieu, L. Praly, and A. Astolfi, "Homogeneous Approximation, Recursive Observer Design, and Output Feedback," SIAM J. Control Optimization, vol. 47, no. 4, pp. 1814-1850, 2008. 